\section{Review}

Correspondence

D. A. Enoch

david.enoch@addenbrookes.

nhs.uk

\title{
Invasive fungal infections: a review of epidemiology and management options
}

\author{
D. A. Enoch, H. A. Ludlam and N. M. Brown \\ Clinical Microbiology and Public Health Laboratory, Health Protection Agency, Addenbrooke's \\ Hospital, Hills Road, Cambridge CB2 2OW, UK
}

\begin{abstract}
Fungi are increasingly recognised as major pathogens in critically ill patients. Candida spp. and Cryptococcus spp. are the yeasts most frequently isolated in clinical practice. The most frequent filamentous fungi (moulds) isolated are Aspergillus spp., but Fusarium spp., Scedosporium spp., Penicillium spp., and Zygomycetes are increasingly seen. Several reasons have been proposed for the increase in invasive fungal infections, including the use of antineoplastic and immunosuppressive agents, broad-spectrum antibiotics, and prosthetic devices and grafts, and more aggressive surgery. Patients with burns, neutropenia, HIV infection and pancreatitis are also predisposed to fungal infection. The epidemiology and clinical features of fungal infections are reviewed, together with antifungal agents currently or soon to be available.
\end{abstract}

\section{Introduction}

Fungi are increasingly recognized as major pathogens in critically ill patients. Candida spp. and Cryptococcus spp. are the yeasts most frequently isolated in clinical practice. The most frequent filamentous fungi (moulds) isolated are Aspergillus spp., but Fusarium spp., Scedosporium spp., Penicillium spp. and Zygomycetes are increasingly seen (Marr et al., 2002; Husain et al., 2003). Several reasons have been proposed for the increase in invasive fungal infections, including the use of antineoplastic and immunosuppressive agents, broad-spectrum antibiotics, and prosthetic devices and grafts, and more aggressive surgery. Patients with burns, neutropenia, HIV infection and pancreatitis are also predisposed to fungal infection (Eggimann et al., 2003).

The epidemiology and clinical features of fungal infections are reviewed, together with antifungal agents currently or soon to be available.

\section{Organisms}

\section{Candida spp.}

Disseminated candidiasis is associated with a mortality in excess of $25 \%$ (Kibbler et al., 2003). Candida is a normal commensal of the skin, and gastrointestinal and genitourinary tracts. Candida albicans is the most frequent species isolated from clinical specimens, but other species (nonalbicans Candida, NAC) are increasingly seen. NAC are of special concern, since some are highly virulent and are associated with treatment failure due to reduced susceptibility to antifungal agents. Although identification to species level and susceptibility testing are technically demanding, it is recommended that they should be performed on all fungi obtained from sterile sites and urine of intensive-care and transplant patients (Denning et al., 2003).
Candidaemia rates increased rapidly in the 1980 s, so that Candida spp. became the fourth-commonest cause of bloodstream infection (BSI) in the USA (Edmond et al., 1999). It has been noted, however, that this increasing trend changed in the late 1990s as a result of a significant decrease in the incidence of C. albicans BSI (Trick et al., 2002). The incidence of NAC BSI remained stable, apart from that due to Candida glabrata, which increased significantly. This rise in C. glabrata also occurred in two prospective studies from Italy (Tortorano et al., 2002, 2004a). Prophylaxis with azole antifungal agents may account for this change in epidemiology. C. albicans was responsible for $79 \cdot 4 \%$ of candidaemias in intensive-care patients, but only $37.5 \%$ in haematology patients (Kibbler et al., 2003). Similar results were seen in a prospective pan-European study (Tortorano et al., 2004b).

Prior surgery, acute renal failure, previous yeast colonization, neutropenia, antibacterial agents, parenteral nutrition and central venous catheters were associated with an increased risk of invasive candidiasis, whilst prior antifungals was protective (Eggimann et al., 2003).

Candida is capable of causing a wide spectrum of disease. Clinical diagnosis of invasive candida infection is challenging: as most symptoms are non-specific, early clinical manifestations are those of sepsis, and cultures may only become positive late in the course of the infection. Late or no treatment are independent predictors of death in invasive candidiasis (Denning et al., 2003).

\section{Cryptococcus spp.}

Cryptococcus neoformans is the commonest cause of cryptococcosis, and is usually acquired by inhalation. Pulmonary cryptococcosis may be asymptomatic or may present 
non-specifically with cough, fever or pleural symptoms. Meningitis is a common feature of infection, especially in HIV-seropositive patients and solid-organ transplant recipients.

A high index of suspicion of cryptococcal infection is needed because of the non-specific clinical findings. A positive serum cryptococcal antigen is reliable for the diagnosis of disseminated disease, and should prompt a lumbar puncture to exclude central nervous system disease, as treatment of cryptococcal meningitis requires more aggressive and prolonged therapy (Saag et al., 2000). All cerebrospinal fluid (CSF) specimens from HIV-seropositive patients, transplant recipients and patients with sarcoidosis that show abnormal biochemical parameters or raised leukocytes without adequate explanation should be tested for cryptococcal antigen and cultures incubated for a prolonged period to encourage isolation of the organism (for example, bacterial cultures kept for a minimum of 5 days and fungal cultures for 21 days) (Denning et al., 2003).

\section{Aspergillus spp.}

Aspergillus spp. are the most commonly isolated invasive moulds (Denning, 1998). Only a few of the 200 or so species are pathogenic to man, primarily Aspergillus fumigatus, Aspergillus flavus and Aspergillus niger.

A. fumigatus remains the mould most frequently isolated, but the epidemiology appears to be changing. A. fumigatus accounted for $82 \%$ of cases of invasive aspergillosis in 1985, compared to $66 \%$ in 1999 in stem cell transplant patients (Marr et al., 2002). Aspergillus terreus is increasingly recognized as a pathogen, accounting for $15 \%$ of isolates in 2001, compared to $<2 \%$ in 1996 in one study (Baddley et al., 2003). Aspergillus spp. can lead to invasive aspergillosis, tracheobronchitis, aspergilloma and chronic necrotising aspergillosis, but colonization without infection can occur.

Risk factors for invasive aspergillosis include prolonged neutropenia (particularly if $>3$ weeks) or neutrophil dysfunction, steroid therapy, haematological malignancy, cytotoxic drugs, AIDS, and transplantation (particularly when a mismatch is present). The risk is pronounced in bone marrow transplantation, where invasive aspergillosis is recorded in up to $13 \%$ of allograft and autograft recipients (Marr et al., 2002). Infliximab, an immunosuppressive monoclonal antibody to TNF- $\alpha$, was recently implicated as a predisposing factor (De Rosa et al., 2003).

Amongst solid-organ transplant patients, lung and heartlung transplant recipients are at greatest risk of infection, which affects $14-18 \%$ of patients (Hagerty et al., 2003). The transplanted lung is at greatest risk, because the respiratory tract is the primary portal of entry, and the lung is denervated below the anastomosis, leading to loss of cough reflexes and mucociliary clearance. Inadequate blood flow, episodes of rejection, and cytomegalovirus (CMV) infection are also implicated. Up to $7 \%$ of liver transplant recipients develop invasive aspergillosis (Hagerty et al., 2003). The diagnosis is typically made between 2 and 4 weeks after transplantation, and is associated with poor allograft function with associated increased immunosuppression/postoperative dialysis. However, more episodes $(55 \%)$ now occur after 3 months, in association with CMV infection (Singh et al., 2003). Invasive aspergillosis occurs in $<1 \%$ of renal transplant cases (Hagerty et al., 2003).

The clinical presentation of invasive aspergillosis varies by patient group, as does the rate at which the disease progresses. As immunosuppression increases, so the rate of progression increases, and the symptoms of infection become less obvious. Invasive aspergillosis may be asymptomatic in up to one-third of patients, and diagnostic difficulties are compounded by the lack of characteristic symptoms and signs. Early symptoms of invasive pulmonary aspergillosis include cough, fever and haemoptysis. Hypoxaemia is usual, and chest radiography changes are variable, although pneumothorax in a neutropenic patient should prompt the clinician to exclude invasive pulmonary aspergillosis as a cause.

Computerized tomography (CT) scanning can be diagnostic (Denning, 1998) and allows early diagnosis. In one study of CT investigation, the mean time to diagnose invasive pulmonary aspergillosis fell from 7 days to $<2$ days, leading to reduced mortality (Caillot et al., 1997). It also aids further diagnostic studies such as bronchoscopy and open lung biopsy, which may lead to improved outcomes. Typical CT signs include multiple nodules, the halo-sign (seen early in disease) and the air-crescent sign, though the latter may occur only when the neutrophil count is recovering (Caillot et al., 2001). Bronchoscopy fluids should be examined microscopically for hyphae and cultured on specialized media. Clinical isolates of aspergilli should be speciated (Denning et al., 2003). Whilst culture of bronchial fluid is specific ( $>95 \%$ ), it lacks sensitivity (30-50\%). Open lung biopsy is considered to be the gold standard, but false negatives can still occur (Denning 1998). Combining CT with aspergillus antigen (galactomannan) detection also shows promise in early diagnosis of invasive pulmonary aspergillosis (Becker et al., 2003). A recent study combining galactomannan detection, CT and early bronchoscopy suggested that pre-emptive rather than empirical therapy may be more appropriate (Maertens et al., 2005). Further studies are required.

Post lung transplant, airway colonization is present in $29 \%$ of cases and subsequent tracheobronchitis occurs frequently, affecting $5 \%$, with the area around the anastamosis most at risk (Mehrad et al., 2001). AIDS patients are also susceptible to tracheobronchitis, including those on effective anti-retroviral therapy.

Cerebral infection may present with headache and focal features (Denning, 1998). Sinusitis can also be due to Aspergillus spp., although this is more commonly seen in haematology patients than in solid-organ transplant recipients. 


\section{Emerging moulds}

Other moulds include Fusarium spp., Scedosporium spp. (hyalohyphomycoses) and Rhizopus spp. (a mucormycosis or zygomycosis). These are capable of causing a wide variety of infections. Most cases of disseminated infection occur in immunocompromised patients, particularly in stem-cell (Marr et al., 2002) and solid-organ (Husain et al., 2003) recipients. Other risk factors include diabetes and renal failure (Ribes et al., 2000).

In haematopoietic stem-cell transplant recipients, the incidence of both Fusarium spp. and Rhizopus spp. infection doubled in the period 1985-1999, whilst that of Scedosporium spp. remained stable (Marr et al., 2002). Overall, these genera accounted for $18 \%$ of all mould infections in this group of patients. Infection with Scedosporium spp. was more likely to occur whilst the patient was still neutropenic, whilst infection with Zygomycetes was more likely to occur later, corresponding to episodes of graft versus host disease (Ribes et al., 2000). One consequence of the use of voriconazole in prophylaxis and treatment has been a rise in the incidence of infections due to Zygomycetes (Marty et al., 2004).

A prospective multi-centre study of mould infections in heart transplant and liver transplant recipients found that Aspergillus spp. were responsible for $69 \%$ of cases of invasive infections (Husain et al., 2003). Scedosporium spp. were responsible for four episodes, Rhizopus spp. and other Zygomycetes for three cases, and there were two cases caused by Fusarium spp. Seven cases were attributed to other mould infections. These moulds were significantly more likely to disseminate from the primary site of infection than Aspergillus spp. and were associated with a higher mortality $(61 \cdot 5$ versus $54 \%$ for aspergillosis). Retransplantation, CMV infection, dialysis and disseminated infection were associated with increased mortality.

\section{Antifungal agents}

\section{Amphotericin B.}

Amphotericin B deoxycholate (AmB-D; Fungizone) is a polyene with a very broad spectrum of activity, including most yeasts and filamentous fungi. Possible exceptions include Candida lusitaniae, Candida guillermondii, Scedosporium spp. and A. terreus.

Side effects are common, occurring in $50-90 \%$ of cases, and are principally nephrotoxicity or infusion-related. Other side effects include fevers, chills, nausea and vomiting, and hypotension. Nephrotoxicity is related to the use of other nephrotoxic agents and diuretics, and the dosage and duration of amphotericin B treatment. Sepsis, hypotension and tissue hypoxia are also risk factors in intensive-care-unit patients. Alternate-day dosing, administration by continuous infusion, combination with flucytosine to allow use of lower doses, and the use of lipid-associated products, have all been employed to overcome these side effects. Eighty patients were randomized to receive AmB-D either over $4 \mathrm{~h}$ or continuously over $24 \mathrm{~h}$ in one trial (Eriksson et al., 2001). Efficacy was equivalent, but nephrotoxicity and infusion-related side effects were significantly lower in the continuous-infusion cohort. However, dedicating an intravenous line for one drug may not be possible in critically ill patients.

Three lipid-associated amphotericin B products are currently available which may have reduced toxicity compared with conventional amphotericin B. These comprise lipid complexes (ABLC-Abelcet, Zeneus Pharma), cholesteryl sulphate (ABCD-Amphocil, Cambridge Laboratories in Europe, Amphotec, InterMune in the USA), and a liposomal formulation (L-AmB; AmBisome, Gilead Sciences). L-AmB is indicated for the treatment of severe systemic and/or deep mycoses in which toxicity (particularly nephrotoxicity) precludes the use of AmB-D in effective dosages, and for the empirical treatment of presumed fungal infections in febrile neutropenic patients, in whom the fever has failed to respond to broad-spectrum antibiotics and appropriate investigations have failed to define a bacterial or viral cause. L-AmB was found to be as effective as AmB-D in the empirical treatment of neutropenic sepsis, and was associated with fewer side effects (Walsh et al., 1999). ABCD is indicated for the treatment of severe systemic and/or deep mycoses in cases in which toxicity or renal failure precludes the use of AmB-D in effective doses and in cases in which prior systemic antifungal therapy has failed. ABCD has been used successfully in severely neutropenic patients. Abelcet is indicated for the treatment of severe invasive candidiasis. Abelcet is also indicated as second-line therapy for the treatment of severe systemic fungal infections in patients who have not responded to AmB-D or other systemic antifungal agents, in those who have renal impairment or other contraindications to AmB-D, or in patients who have developed amphotericin B nephrotoxicity. Abelcet treatment is indicated as second-line treatment for invasive aspergillosis, cryptococcal meningitis and disseminated cryptococcosis in HIV patients, fusariosis, coccidiomycosis, zygomycosis and blastomycosis. L-AmB given at $5 \mathrm{mg} \mathrm{kg}^{-1}$ demonstrated higher efficacy than AmB-D in a study of patients with invasive aspergillosis (Leenders et al. 1998), whilst a meta-analysis suggested that lipid-associated formulations reduced all-cause mortality for invasive fungal infections by almost $30 \%$ compared with AmB-D (Barrett et al., 2003). Higher treatment doses of amphotericin B were tolerated due to a reduction in nephrotoxicity. Other drugs with dose-limiting nephrotoxicity (e.g. ciclosporin) may be better tolerated, thereby improving clinical outcome. The meta-analysis failed to show any advantage in outcome of any one lipid-based formulation over another. However, assuming clinical outcome is related to tolerability, the differences in tolerability between the agents assume greater importance. In comparative studies, adverse events requiring discontinuation of the drug have been least with liposomal L-AmB (12\%), intermediate with ABLC $(32 \%)$ and highest with $\mathrm{ABCD}(41 \%)$, the latter figure being comparable to that of AmB-D (44\%) (White et al., 
1998; Wingard et al., 2000). The use of lipid-associated formulations is often restricted, because of expense, to patients at high risk of side effects, those who are intolerant of AmB-D, or those who require high doses for prolonged periods.

\section{Flucytosine}

Flucytosine (Ancotil, Valeant) is licensed for use in the treatment of systemic fungal infections caused by sensitive organisms. It has activity against Candida spp., Cryptococcus spp. and some filamentous fungi. Intrinsic resistance in Candida spp. is uncommon (Ostrosky-Zeichner et al., 2003), but flucytosine must be combined with another antifungal agent because of the rapid emergence of resistance when used alone. Drug concentrations must be measured regularly, especially with prolonged use of high doses, or when combined with nephrotoxic agents. Side effects are dose-dependent and often predictable, and are rare if peak levels are kept below $100 \mathrm{mg} \mathrm{l}^{-1}$ (Vermes et al., 2000). Gastrointestinal side effects (nausea and diarrhoea), hepatotoxicity and bone marrow suppression are reversible on discontinuation of the drug. They are associated with peak levels greater than $125 \mathrm{mg} \mathrm{l}^{-1}$ (Vermes et al., 2000). Flucytosine levels are reduced by cytarabine, and caution must be employed if used with other myelosuppressive drugs. The combination of amphotericin B with flucytosine has been shown to be beneficial in randomized controlled trials of patients with cryptococcal meningitis (Bennett et al., 1979) and its use is recommended (Saag et al., 2000). Penetration of the drug into the eye and central nervous system is good. Its use is recommended, in combination, in patients with endophthalmitis, meningitis and endocarditis due to Candida spp. (Pappas et al., 2004).

\section{Azoles}

\section{Fluconazole}

Fluconazole (Diflucan, Pfizer) is available in oral or intravenous formulations and is well tolerated, with fewer drug interactions than other azoles. It is inactive against filamentous fungi. Fluconazole has been shown to be as effective as amphotericin B in the treatment of candidaemia in nonneutropenic patients in two large randomized controlled trials (Rex et al., 1994; Phillips et al., 1997). Approximately $5 \%$ of isolates of C. albicans were resistant to fluconazole, whilst resistance to fluconazole in isolates of Candida krusei and C. glabrata was noted in $34 \%$ (of 50) and $8 \%$ (of 458) isolates, respectively (Ostrosky-Zeichner et al., 2003). No resistance, however, occurred in 23 isolates of C. glabrata in another study (Kibbler et al., 2003). Fluconazole may be inappropriate for the empirical treatment of suspected fungal infection in neutropenic patients, because prior exposure, as treatment or prophylaxis, is associated with resistant candidal strains, and because of its lack of activity against moulds (Pappas et al., 2004). It may be useful, however, in selected patients who have not received prior azoles, have no risk factors for or features of invasive aspergillosis, and in whom it is unlikely that an azole-resistant
Candida spp. is responsible for infection (Winston et al., 1999).

\section{Itraconazole}

Itraconazole (Sporanox, Janssen-Cilag) has a wider spectrum than fluconazole. It is active against yeasts and moulds, with the exception of Fusarium spp., Scedosporium spp. and the Zygomycetes (Johnson et al., 1998). Reduced susceptibilities occurred in $18 \%$ of bloodstream isolates in the USA ( $8 \%$ of C. albicans and $51 \%$ of C. glabrata) (OstroskyZeichner et al., 2003). It has unpredictable bioavailability in capsule form and therapeutic drug monitoring is recommended (Stevens et al., 2000). The introduction of an intravenous preparation and a well-absorbed oral solution has overcome this problem (Barone et al., 1998; Slain et al., 2001).

Clinical data regarding the efficacy of intravenous itraconazole, and information regarding the treatment of invasive candidiasis and invasive aspergillosis, is limited. Itraconazole has been compared with AmB-D in the empirical treatment of neutropenic sepsis (Boogaerts et al., 2001). Mortality rates were similar, whilst itraconazole was associated with significantly fewer adverse events ( 5 versus $54 \%$ ), and significantly less withdrawal because of adverse events (19 versus $38 \%$ ) and nephrotoxicity (5 versus $24 \%$ ). Intravenous itraconazole is approved for the treatment of pulmonary and extrapulmonary aspergillosis in patients who are intolerant of or who have infections refractory to amphotericin B (Slain et al., 2001). A small retrospective analysis of haematological patients with invasive aspergillosis reported no difference in outcome for patients treated with either amphotericin B or itraconazole (Denning et al., 1998). It is therefore considered acceptable to use itraconazole $\left(400 \mathrm{mg} \mathrm{day}^{-1}\right.$ ) as intravenous or oral follow-on therapy after initial amphotericin B for the treatment of invasive aspergillosis (Stevens et al., 2000). Itraconazole has been compared with fluconazole for prophylaxis in patients receiving allogenic stem-cell transplants. One study showed no difference (Morgenstern et al., 1999), whilst another showed a reduction in invasive mould infections but no change in mortality at 250 days (Marr et al., 2004). Itraconazole was associated with significantly more side effects leading to discontinuation (36 versus $16 \%$ ). These findings were echoed in a recent meta-analysis (Vardakas et al. 2005). Side effects included gastrointestinal symptoms, headache, dizziness, raised hepatic transaminases, menstrual disorders, peripheral neuropathy and allergic reactions. Heart failure has been reported, and the UK Committee on Safety of Medicines has advised caution when prescribing itraconazole to older patients or those with cardiac disease. This is particularly important in patients on high doses for prolonged periods.

Itraconazole is known to have many interactions with other drugs which critically ill and immunosuppressed patients are likely to be receiving. Itraconazole is mainly metabolized through the cytochrome CYP3A4. The anticoagulant effect 
of warfarin is enhanced by itraconazole. Plasma levels of tacrolimus, ciclosporin, protease inhibitors, statins, calcium channel blockers, digoxin, quinidine, carbamazepine and pimozide are all increased. Itraconazole decreases plasma concentrations of isoniazid, rifampicin, rifabutin, nevirapine and phenytoin, whilst itraconazole levels can be increased by co-administration of macrolides and protease inhibitors.

\section{Voriconazole}

Voriconazole (Vfend, Pfizer) has a spectrum similar to that of itraconazole, but extending to several emerging moulds, including Fusarium spp. and Scedosporium spp. (Diekema et al., 2003). It has in vitro activity against many fluconazoleresistant Candida spp. (Pfaller et al., 2003). It is licensed in Europe for the treatment of fluconazole-resistant invasive Candida infection. Voriconazole was found to be noninferior to AmB-D followed by fluconazole in a recent randomized trial of non-neutropenic candidaemia when using a 12-week end point (Kullberg et al., 2005). The success rate at the end of therapy was higher $(71 \%)$ with the AmB-D/fluconazole arm than the voriconazole arm (66\%). Adverse reactions were more common in the AmBD/fluconazole arm, but treatment discontinuation due to adverse events was higher in the voriconazole arm. No trials have been published comparing voriconazole to L-AmB, fluconazole alone or caspofungin in candidaemia. Voriconazole has been compared with fluconazole in the treatment of oesophageal candidiasis, in which it was as effective but was less well tolerated (Ally et al., 2001). More patients discontinued voriconazole because of laboratory test abnormalities and treatment-related adverse events. Voriconazole is also licensed for the primary treatment of invasive aspergillosis and has been compared with AmB-D (1.0-1.5 mg $\mathrm{kg}^{-1}$ day $^{-1}$ ) in a non-blinded trial (Herbrecht et al., 2002). Significantly more patients experienced adverse events with amphotericin B (421 events) than with voriconazole (343 events), but no data were presented for the discontinuation rates. At week 12, the survival rate for the voriconazole arm was $71 \%$, and $58 \%$ for the amphotericin B arm in the modified intention to treat population. However, the data to suggest that it is superior to amphotericin B are questionable. There were differences in the duration of the initial, non-blinded treatment between voriconazole and amphotericin $\mathrm{B}$, and data indicating superiority are derived from subgroup analysis ( $71 \%$ of all randomized patients) of a secondary outcome measure (survival). A non-blinded trial has compared voriconazole with liposomal amphotericin B in neutropenic patients who remained febrile despite $96 \mathrm{~h}$ of therapy with antibacterial agents (Walsh et al., 2002). The rates of successful outcome did not differ between the two groups (26 versus $30 \cdot 6 \%$, respectively), and both were equally well tolerated. However, significantly more stopped voriconazole therapy due to lack of efficacy $(5 \cdot 3$ versus $1 \cdot 2 \%$, respectively), principally due to persistent fever. Voriconazole is not approved for empirical treatment of febrile neutropenia because the composite primary end points showing superiority were not reached in the study.
Non-inferiority compared with liposomal amphotericin B was therefore not excluded.

Voriconazole is licensed for the treatment of infections due to Scedosporium spp. and Fusarium spp. on the basis of several case reports. It is ineffective in vitro against isolates of Zygomycetes (Sun et al., 2002), and its use may be associated with increased isolation rates of these organisms (Marty et al., 2004). Increased MICs have been seen in an isolate from a patient previously treated with fluconazole (Tortorano et al., 2004b). Although voriconazole has in vitro activity against Cryptococcus spp., no clinical trials have been published to show superiority over fluconazole, and it remains unlicensed for this indication. Voriconazole is available in oral and intravenous forms. Visual disturbances have been reported in $45 \%$ of patients with voriconazole in clinical trials (Herbrecht et al., 2002). Severe reactions, such as Stevens Johnson syndrome, toxic epidermal necrolysis, pancreatitis, hepatitis and jaundice, have been described. Liver function should be monitored before treatment is commenced, and then at 2-4-week intervals. Less-severe side effects include fever, gastrointestinal symptoms, headache and hypotension (Johnson \& Kauffman, 2003). Voriconazole is metabolized by cytochrome $\mathrm{P} 450$ isoenzymes CYP2C19, CYP2C9 and CYP3A4, and therefore has many drug interactions, similar to those of itraconazole.

\section{Ravuconazole and posaconazole}

Ravuconazole and posaconazole have a spectrum of activity similar to that of voriconazole, and are currently undergoing phase III trials. Posaconazole is available only in an oral preparation, but its use has been described in case reports in the treatment of Scedosporium spp. (Mellinghoff et al., 2002) and zygomycosis (Tobon et al., 2003). In in vitro studies, the activity of posaconazole and ravuconazole was equivalent to that of voriconazole and amphotericin B against Candida spp. (Laverdiere et al., 2002; Pfaller et al., 2003) and moulds (Diekema et al., 2003). Posaconazole had more activity in vitro against Zygomycetes than voriconazole (Sun et al., 2002), and was found to be more active than amphotericin B against Scedosporium spp. (Carrillo \& Guarro, 2001). Reduced susceptibility to posaconazole, developing on treatment in an HIV-infected individual, leading to treatment failure, has already been shown to develop in a clinical isolate of C. albicans (Li et al., 2004). Posaconazole has been reviewed recently (Torres et al., 2005).

\section{Echinocandins \\ Caspofungin}

The spectrum of caspofungin (Merck Sharpe \& Dohme) is limited to Candida spp., Saccharomyces spp., Pneumocystis carinii and Aspergillus spp., including A. terreus (Denning, 2003). It has good activity against C. glabrata, but Candida parapsilosis may respond less readily to treatment (Pappas et al., 2004). Caspofungin is available for intravenous use only.

Caspofungin is licensed for the treatment of invasive candidiasis in non-neutropenic adults. Caspofungin was 
as effective as AmB-D in the treatment of candidaemia, but was better tolerated (Mora-Duarte et al., 2002). Caspofungin was as effective as amphotericin $\mathrm{B}\left(0.5 \mathrm{mg} \mathrm{kg}^{-1} \mathrm{day}^{-1}\right)$ (Villanueva et al., 2001) and fluconazole (200 mg) (Villanueva et al., 2002) in the treatment of patients with oesophageal candidiasis, and was well tolerated. Caspofungin is also licensed for the treatment of invasive aspergillosis in adult patients who are refractory to or intolerant of AmB-D, lipid formulations of amphotericin B and/or itraconazole. Refractoriness is defined as progression of infection or failure to improve after a minimum of 7 days of prior therapeutic doses of effective antifungal therapy. A favourable response was noted in $45 \%$ of 83 patients (Maertens et al., 2004). Caspofungin is also licensed for empirical therapy for presumed fungal infections (such as Candida or Aspergillus) in febrile neutropenic adults. Caspofungin was compared to liposomal amphotericin B in a randomized controlled trial involving over 1000 neutropenic patients, and was found to be as effective, but generally better tolerated, in this group of patients (Walsh et al., 2004). Patients on ciclosporin were excluded from this study.

Side effects are uncommon and usually mild. The most important are fever, phlebitis, rash and chills. Mild elevations in liver transaminases and electrolyte disturbances have also been noticed (Mora-Duarte et al., 2002). Headaches have been described (Villanueva et al., 2001, 2002). There are a number of potentially important drug interactions. Rifampicin can reduce caspofungin blood concentrations after prompting an initial rise, whereas ciclosporin may cause a sustained rise in caspofungin levels and area under the curve. Monitoring liver function is advised. Safety data for dual therapy is limited to animal data and small case reports. Increases in caspofungin clearance have been noted when caspofungin is given with carbamazepine, dexamethasone, efavirenz, nelfinavir, nevirapine and phenytoin, suggesting modest dosage increases in caspofungin may be advisable (Denning, 2003). Caspofungin can cause blood concentrations of tacrolimus to fall, and monitoring of tacrolimus levels is advised.

Anidulafungin and micafungin are two new echinocandin agents under development for the treatment of Candida spp. Open-label trials have been published with micafungin (Ostrosky-Zeichner et al., 2005), but randomized trials are required.

\section{Combination therapy}

Potential benefits of combination therapy include enhanced potency of antifungal efficacy, reduced selection of resistant organisms (particularly for flucytosine), and reduced toxicities due to lower dosing. Evidence of benefit is clearest for the treatment of cryptococcal meningitis, in which the combination of AmB-D and flucytosine leads to earlier sterilization of the CSF (Bennett et al., 1979) and reduced relapse rates (Saag et al., 1999). In vitro antagonism of anticandidal activity has been reported for amphotericin B and azoles, and this combination was not recommended for the treatment of candidal infections (British Society for Antimicrobial Chemotherapy Working Party, 1994). However, no antagonism was noted clinically in a randomized and blinded trial comparing amphotericin $\mathrm{B}\left(0.7 \mathrm{mg} \mathrm{kg}^{-1}\right.$ day $\left.^{-1}\right)$ plus fluconazole $\left(800 \mathrm{mg}\right.$ day $\left.{ }^{-1}\right)$ with amphotericin B alone (Rex et al., 2003) for the treatment of candidaemia in non-neutropenic patients. Amphotericin B plus fluconazole showed faster bloodstream sterilization than amphotericin $\mathrm{B}$ alone.

Despite the high mortality of invasive aspergillosis, there are no published trials of combination therapy for its treatment. In case reports, AmB-D has been combined with flucytosine with occasional success (Darras-Joly et al., 1996). The combination of azoles with amphotericin B, however, is often used. No prospective studies examine the crossover effect from amphotericin B to itraconazole, despite the sequential use of these agents being in the recommendations for the treatment of aspergillosis (Stevens et al., 2000). The sequential use of an azole followed by amphotericin B has also not been studied extensively. A review of published in vitro and in vivo interactions and 6281 clinical cases revealed only 18 patients treated with itraconazole followed by amphotericin B, with generally poor results (in some instances antagonism was demonstrated) and no standardization (Steinbach et al., 2003). In vitro studies of caspofungin with azoles or amphotericin B have generally shown indifference or synergy. Clinical data are limited at present to case reports of patients with invasive aspergillosis. Caspofungin has been used with itraconazole in the treatment of a patient with acute lymphoblastic leukaemia who had invasive pulmonary aspergillosis due to $A$. terreus. The patient remained free of Aspergillus infection for more than 7 months, but died of her underlying condition. In another case at the same institution, a lung transplant recipient with aspergillosis due to A. fumigatus did not tolerate itraconazole combined with ABLC (5 $\mathrm{mg} \mathrm{kg}^{-1}$ daily), and so the ABLC was switched to caspofungin, which was well tolerated, the renal function returning to normal (Rubin et al., 2002). In an uncontrolled retrospective report of 48 patients, caspofungin was added to liposomal amphotericin B as salvage therapy in cases of invasive aspergillosis refractory to the latter agent. The combination was well tolerated (Kontoyiannis et al., 2003).

\section{Prophylaxis}

The use of fluconazole prophylaxis $\left(400 \mathrm{mg} \mathrm{day}{ }^{-1}\right)$ is commonplace in haematology units, and has been shown to reduce the incidence and severity of fungal infections (Goodman et al., 1992; Slavin et al., 1995) when compared to placebo in bone marrow transplant patients. Metaanalysis has shown improved mortality in stem-cell transplant recipients and patients treated for acute leukaemia (Cornely et al., 2003). Long-term follow-up of stem-cell transplant patients revealed that the persistent protection (i.e. beyond the period of engraftment) in those given fluconazole was beneficial (Marr et al., 2000). Itraconazole $\left(2.5 \mathrm{mg} \mathrm{kg}^{-1}\right.$ twice daily) has been compared to 
fluconazole in this group of patients and found to be comparable in preventing candidal infection but more effective in preventing mould infection (Morgenstern et al., 1999). A recent trial, however, showed itraconazole to be more toxic and less well tolerated than fluconazole, with no improvement in mortality (Marr et al., 2004). The duration of prophylaxis is currently unknown, but it has been recommended that this should cover the period of neutropenia (Pappas et al., 2004).

Antifungal prophylaxis in the intensive-care setting has been studied extensively, with mixed results. The most recent guidelines suggest that fluconazole may be considered in carefully selected patients if high rates of invasive candidiasis persist despite standard infection-control procedures (Pappas et al., 2004). Patients with severe acute pancreatitis may benefit from early fluconazole prophylaxis (De Waele et al., 2003).

The value of prophylaxis against invasive aspergillus infection remains uncertain. Aerosolized amphotericin B (10 mg twice daily) was compared with no prophylaxis in a randomized trial, but no differences in mortality were noted, and it was poorly tolerated (Schwartz et al., 1999). Risk factors for invasive fungal infection in liver transplant patients include retransplantation, raised creatinine, choledochojejunostomy, significant blood transfusion and fungal colonization (Hagerty et al., 2003). The incidence of invasive Candida infection in these patients was reduced by prophylactic fluconazole (compared with placebo), but without effect on mortality (Winston et al., 1999).

Oesophageal candidiasis in HIV-seropositive patients and patients with cancer can be prevented with long-term suppressive therapy with fluconazole. Fluconazole (200 mg daily) has been used as maintenance therapy in HIVseropositive patients who experienced an episode of cryptococcal meningitis. This was compared favourably to itraconazole (200 mg daily). Relapse occurred in $4 \%$ of the fluconazole-treated arm and $23 \%$ of the itraconazoletreated arm (Saag et al., 1999).

The uncertain prophylactic value of antifungals and the concern surrounding the generation of resistance prompted the Infectious Disease Society of America to recommend against their general use in neutropenic patients with cancer (Hughes et al., 2002). In view of these uncertainties, it should be noted that careful observation of standard infectioncontrol policies, such as hand washing, use of positive pressure and HEPA-filtered airflow, observation of guidelines for catheter insertion and care, and antibiotic control, are all associated with lowering the incidence of fungal infections in critically ill patients (Manuel \& Kibbler, 1998).

\section{Adjunctive therapies}

Removal of intravascular cannulae is recommended in the treatment of candidaemias whenever possible, as they are the usual portal of entry (Pappas et al., 2004). Overall mortality from candidaemia following cannula removal was $40 \%$, compared with $86.9 \%$ when cannulae were not removed (Kibbler et al., 2003). Evidence for benefit of line removal is strongest in non-neutropenic patients. The gut is a likely source of infection in neutropenic patients. Treatment of endogenous Cushing's syndrome or lowering doses of iatrogenic corticosteroids is associated with improved outcome.

Several recombinant cytokines have been used in patients at risk of fungal infections. Shortening the period of neutropenia shortens the period of greatest risk, and can be achieved by granulocyte-colony stimulating factor (G-CSF) and granulocyte monocyte-colony stimulating factor (GMCSF). Interferon $\gamma$, interleukin (IL)-12 and anti-IL-4 may be protective as these enhance Th-1-dependent immunity, which is also important in host defence against invasive fungal infections (Roilides et al., 1998).

\section{Conclusions}

Amphotericin B, fluconazole and itraconazole are the cornerstones of treatment of invasive fungal infections. The toxicity of AmB-D is frequently dose limiting, and this problem can be avoided with lipid-associated formulations. Voriconazole and caspofungin are two drugs recently licensed for the treatment of invasive fungal infections. Voriconazole has a broad spectrum of activity and is available both orally and parenterally, and may be suitable as second-line therapy in selected patients, particularly those infected with unusual organisms resistant to first-line agents. However, there is no convincing evidence that it is superior to amphotericin B or itraconazole in the treatment of invasive candidal or aspergillus infections. Caspofungin is available in intravenous form only and appears to be better tolerated than AmB-D. At present it is used mainly as a second-line agent in patients with life-threatening invasive candidal or aspergillus infections unresponsive to first-line therapy.

These are interesting times for those managing invasive fungal infections. The increasing numbers of increasingly immunosuppressed patients has led to a rising burden of fungal infection, and the widespread use of antifungal prophylaxis is changing the pattern of fungi recovered in favour of resistant organisms. Pharmaceutical companies are responding to this challenge with an increasing stock of antifungal and immunomodulating agents. Further welldesigned studies are needed urgently to guide the use of these drugs.

\section{Acknowledgements}

D. A. E. has no conflicts of interest. H. A. L. is in receipt of educational grants from Gilead Sciences International, Janssen-Cilag, Merck Sharpe \& Dohme and Pfizer. N. M. B. has sat on an advisory committee for Merck Sharpe \& Dohme, and has received lecture honoraria from Gilead Sciences International, Merck Sharpe \& Dohme and Pfizer. 


\section{References}

Ally, R., Schurmann, D., Kreisel, W., Carosi, G., Aguirrebengoa, K., Dupont, B., Hodges, M., Troke, P. \& Romero, A. J., and the Esophageal Candidiasis Study Group (2001). A randomized, double-blind, double-dummy, multicenter trial of voriconazole and fluconazole in the treatment of esophageal candidiasis in immunocompromised patients. Clin Infect Dis 33, 1447-1454.

Baddley, J. W., Pappas, P. G., Smith, A. C. \& Moser, S. A. (2003). Epidemiology of Aspergillus terreus at a university hospital. J Clin Microbiol 41, 5525-5529.

Barone, J. A., Moskovitz, B. L., Guarnieri, J., Hassell, A. E., Colaizzi, J. L., Bierman, R. H. \& Jessen, L. (1998). Enhanced bioavailability of itraconazole in hydroxypropyl-beta-cyclodextrin solution versus capsules in healthy volunteers. Antimicrob Agents Chemother 42, 1862-1865.

Barrett, J. P., Vardulaki, K. A., Conlon, C. \& 10 other authors (2003). A systematic review of the antifungal effectiveness and tolerability of amphotericin B formulations. Clin Ther 25, 1295-1320.

Becker, M. J., Lugtenburg, E. J., Cornelissen, J. J., Van Der Schee, C., Hoogsteden, H. C. \& De Marie, S. (2003). Galactomannan detection in computerized tomography-based broncho-alveolar lavage fluid and serum in haematological patients at risk for invasive pulmonary aspergillosis. Br J Haematol 121, 448-457.

Bennett, J. E., Dismukes, W. E., Duma, R. J. \& 12 other authors (1979). A comparison of amphotericin $B$ alone and combined with flucytosine in the treatment of cryptoccal meningitis. $N$ Engl J Med 301, 126-131.

Boogaerts, M., Winston, D. J., Bow, E. J. \& 7 other authors, and the Itraconazole Neutropenia Study Group (2001). Intravenous and oral itraconazole versus intravenous amphotericin B deoxycholate as empirical antifungal therapy for persistent fever in neutropenic patients with cancer who are receiving broad-spectrum antibacterial therapy. A randomized, controlled trial. Ann Intern Med 135, 412-422.

British Society for Antimicrobial Chemotherapy Working Party (1994). Management of deep Candida infection in surgical and intensive care unit patients. British Society for Antimicrobial Chemotherapy Working Party. Intensive Care Med 20, 522-528.

Caillot, D., Casasnovas, O., Bernard, A. \& 10 other authors (1997). Improved management of invasive pulmonary aspergillosis in neutropenic patients using early thoracic computed tomographic scan and surgery. J Clin Oncol 15, 139-147.

Caillot, D., Couaillier, J. F., Bernard, A. \& 8 other authors (2001). Increasing volume and changing characteristics of invasive pulmonary aspergillosis on sequential thoracic computed tomography scans in patients with neutropenia. J Clin Oncol 19, 253-259.

Carrillo, A. J. \& Guarro, J. (2001). In vitro activities of four novel triazoles against Scedosporium spp. Antimicrob Agents Chemother 45, 2151-2153.

Cornely, O. A., Ullmann, A. J. \& Karthaus, M. (2003). Evidence-based assessment of primary antifungal prophylaxis in patients with hematologic malignancies. Blood 101, 3365-3372.

Darras-Joly, C., Veber, B., Bedos, J. P., Gachot, B., Regnier, B. \& Wolff, M. (1996). Nosocomial cerebral aspergillosis: a report of 3 cases. Scand J Infect Dis 28, 317-319.

Denning, D. W. (1998). Invasive aspergillosis. Clin Infect Dis 26, 781-803.

Denning, D. W. (2003). Echinocandin antifungal drugs. Lancet 362, 1142-1151.

Denning, D. W., Marinus, A., Cohen, J. \& 11 other authors (1998). An EORTC multicentre prospective survey of invasive aspergillosis in haematological patients: diagnosis and therapeutic outcome. EORTC Invasive Fungal Infections Cooperative Group. J Infect 37, 173-180.

Denning, D. W., Kibbler, C. C. \& Barnes, R. A., and on behalf of the British Society for Medical Mycology (2003). British Society for Medical Mycology proposed standards of care for patients with invasive fungal infections. Lancet Infect Dis 3, 230-240.

De Rosa, F. G., Shaz, D., Campagna, A. C., Dellaripa, P. E., Khettry, U. \& Craven, D. E. (2003). Invasive pulmonary aspergillosis soon after therapy with infliximab, a tumor necrosis factor-alphaneutralizing antibody: a possible healthcare-associated case? Infect Control Hosp Epidemiol 24, 477-482.

De Waele, J. J., Vogelaers, D., Blot, S. \& Colardyn, F. (2003). Fungal infections in patients with severe acute pancreatitis and the use of prophylactic therapy. Clin Infect Dis 37, 208-213.

Diekema, D. J., Messer, S. A., Hollis, R. J., Jones, R. N. \& Pfaller, M. A. (2003). Activities of caspofungin, itraconazole, posaconazole, ravuconazole, voriconazole, and amphotericin B against 448 recent clinical isolates of filamentous fungi. J Clin Microbiol 41, 3623-3626.

Edmond, M. B., Wallace, S. E., McClish, D. K., Pfaller, M. A., Jones, R. N. \& Wenzel, R. P. (1999). Nosocomial bloodstream infections in United States hospitals: a three-year analysis. Clin Infect Dis 29, 239-244.

Eggimann, P., Garbino, J. \& Pittet, D. (2003). Epidemiology of Candida species infections in critically ill non-immunosuppressed patients. Lancet Infect Dis 3, 685-702.

Eriksson, U., Seifert, B. \& Schaffner, A. (2001). Comparison of effects of amphotericin B deoxycholate infused over 4 or 24 hours: randomised controlled trial. BMJ 322, 579-582.

Goodman, J. L., Winston, D. J., Greenfield, R. A. \& 7 other authors (1992). A controlled trial of fluconazole to prevent fungal infections in patients undergoing bone marrow transplantation. $N$ Engl J Med 326, 845-851.

Hagerty, J. A., Ortiz, J., Reich, D. \& Manzarbeitia, C. (2003). Fungal infections in solid organ transplant patients. Surg Infect (Larchmont) 4, 263-271.

Herbrecht, R., Denning, D. W., Patterson, T. F. \& 19 other authors, for the Invasive Fungal Infections Group of the European Organisation for Research and Treatment of Cancer and the Global Aspergillus Study Group (2002). Voriconazole versus amphotericin B for primary therapy of invasive aspergillosis. $N$ Engl J Med 347, 408-415.

Hughes, W. T., Armstrong, D., Bodey, G. P. \& 8 other authors (2002). 2002 guidelines for the use of antimicrobial agents in neutropenic patients with cancer. Clin Infect Dis 34, 730-751.

Husain, S., Alexander, B. D., Munoz, P. \& 12 other authors (2003) Opportunistic mycelial fungal infections in organ transplant recipients: emerging importance of non-Aspergillus mycelial fungi. Clin Infect Dis 37, 221-229.

Johnson, L. B. \& Kauffman, C. A. (2003). Voriconazole: a new triazole antifungal agent. Clin Infect Dis 36, 630-637.

Johnson, E. M., Szekely, A. \& Warnock, D. W. (1998). In-vitro activity of voriconazole, itraconazole and amphotericin B against filamentous fungi. J Antimicrob Chemother 42, 741-745.

Kibbler, C. C., Seaton, S., Barnes, R. A., Gransden, W. R., Holliman, R. E., Johnson, E. M., Perry, J. D., Sullivan, D. J. \& Wilson, J. A (2003). Management and outcome of bloodstream infections due to Candida species in England and Wales. J Hosp Infect 54, 18-24.

Kontoyiannis, D. P., Hachem, R., Lewis, R. E. \& 7 other authors (2003). Efficacy and toxicity of caspofungin in combination with liposomal amphotericin B as primary or salvage treatment of invasive aspergillosis in patients with hematologic malignancies. Cancer 98, 292-299. 
Kullberg, B. J., Sobel, J. D., Ruhnke, M. \& 11 other authors (2005). Voriconazole versus a regimen of amphotericin B followed by fluconazole for candidaemia in non-neutropenic patients: a randomised non-inferiority trial. Lancet 366, 1435-1442.

Laverdiere, M., Hoban, D., Restieri, C. \& Habel, F. (2002). In vitro activity of three new triazoles and one echinocandin against Candida bloodstream isolates from cancer patients. J Antimicrob Chemother 50, 119-123.

Leenders, A. C., Daenen, S., Jansen, R. L. H. \& 9 other authors (1998). Liposomal amphotericin B compared with amphotericin B deoxycholate in the treatment of documented and suspected neutropenia-associated invasive fungal infections. $\mathrm{Br} \mathrm{J}$ Haematol 103, 205-212.

Li, X., Brown, N., Chau, A. S., Lopez-Ribot, J. L. \& 7 other authors (2004). Changes in susceptibility to posaconazole in clinical isolates of Candida albicans. J Antimicrob Chemother 53, 74-80.

Maertens, J., Raad, I., Petrikkos, G. \& 10 other authors, for the Caspofungin Salvage Aspergillosis Study Group (2004). Efficacy and safety of caspofungin for treatment of invasive aspergillosis in patients refractory to or intolerant of conventional antifungal therapy. Clin Infect Dis 39, 1563-1571.

Maertens, J., Theunissen, K., Verhof, G. \& 7 other authors (2005). Galactomannan and computed tomography-based preemptive antifungal therapy in neutropenic patients at high risk for invasive fungal infection: a prospective feasibility study. Clin Infect Dis 41, 1242-1250.

Manuel, R. J. \& Kibbler, C. C. (1998). The epidemiology and prevention of invasive aspergillosis. J Hosp Infect 39, 95-109.

Marr, K. A., Seidel, K., Slavin, M. A., Bowden, R. A., Schoch, H. G., Flowers, M. E., Corey, L. \& Boeckh, M. (2000). Prolonged fluconazole prophylaxis is associated with persistent protection against candidiasis-related death in allogeneic marrow transplant recipients: long-term follow-up of a randomized, placebo-controlled trial. Blood 96, 2055-2061.

Marr, K. A., Carter, R. A., Crippa, F., Wald, A. \& Corey, L. (2002). Epidemiology and outcome of mould infections in hematopoietic stem cell transplant recipients. Clin Infect Dis 34, 909-917.

Marr, K. A., Crippa, F., Leisenring, W., Hoyle, M., Boeckh, M., Balajee, S. A., Nichols, W. G., Musher, B. \& Corey, L. (2004). Itraconazole versus fluconazole for prevention of fungal infections in patients receiving allogeneic stem cell transplants. Blood 103, $1527-1533$.

Marty, F. M., Cosimi, L. A. \& Baden, L. R. (2004). Breakthrough zygomycosis after voriconazole treatment in recipients of hematopoietic stem-cell transplants. N Engl J Med 350, 950-952.

Mehrad, B., Paciocco, G., Martinez, F. J., Ojo, T. C., lannettoni, M. D. \& Lynch, J. P., 3rd (2001). Spectrum of Aspergillus infection in lung transplant recipients: case series and review of the literature. Chest 119, 169-175.

Mellinghoff, I. K., Winston, D. J., Mukwaya, G. \& Schiller, G. J. (2002). Treatment of Scedosporium apiospermum brain abscesses with posaconazole. Clin Infect Dis 34, 1648-1650.

Mora-Duarte, J., Betts, R., Rotstein, C. \& 7 other authors, for the Caspofungin Invasive Candidiasis Study Group (2002). Comparison of caspofungin and amphotericin B for invasive candidiasis. N Engl J Med 347, 2020-2029.

Morgenstern, G. R., Prentice, A. G., Prentice, H. G., Ropner, J. E., Schey, S. A. \& Warnock, D. W., on behalf of the UK Multicentre Antifungal Prophylaxis Study Group (1999). A randomized controlled trial of itraconazole versus fluconazole for the prevention of fungal infections in patients with haematological malignancies. Br J Haematol 105, 901-911.
Ostrosky-Zeichner, L., Rex, J. H., Pappas, P. G. \& 9 other authors (2003). Antifungal susceptibility survey of 2,000 bloodstream Candida isolates in the United States. Antimicrob Agents Chemother 47, 3149-3154.

Ostrosky-Zeichner, L., Kontoyiannis, D. P., Raffalli, J. \& 10 other authors (2005). International, open-label, noncomparative, clinical trial of micafungin alone and in combination for treatment of newly diagnosed and refractory candidemia. Eur J Clin Microbiol Infect Dis 24, 654-661.

Pappas, P. G., Rex, J. H., Sobel, J. D., Filler, S. G., Dismukes, W. E., Walsh, T. J. \& Edwards, J. E., on behalf of the Infectious Diseases Society of America (2004). Guidelines for treatment of candidiasis. Clin Infect Dis 38, 161-189.

Pfaller, M. A., Diekema, D. J., Messer, S. A., Boyken, L., Hollis, R. J. \& Jones, R. N., and the International Fungal Surveillance Participant Group (2003). In vitro activities of voriconazole, posaconazole, and four licensed systemic antifungal agents against Candida species infrequently isolated from blood. J Clin Microbiol 41, 78-83.

Phillips, P., Shafran, S., Garber, G. \& 9 other authors, and for the Canadian Candidemia Study Group (1997). Multicenter randomized trial of fluconazole versus amphotericin B for treatment of candidemia in non-neutropenic patients. Eur J Clin Microbiol Infect Dis 16, 337-345.

Rex, J. H., Bennett, J. E., Sugar, A. M. \& 9 other authors, for the Candidemia Study Group and the National Institute of Allergy and Infectious Diseases Mycoses Study Group (1994). A randomized trial comparing fluconazole with amphotericin B for the treatment of candidemia in patients without neutropenia. $N$ Engl J Med 331, 1325-1330.

Rex, J. H., Pappas, P. G., Karchmer, A. W. \& 32 other authors, for the National Institute of Allergy and Infectious Diseases Mycoses Study Group (2003). A randomized and blinded multicenter trial of high-dose fluconazole plus placebo versus fluconazole plus amphotericin B as therapy for candidemia and its consequences in nonneutropenic subjects. Clin Infect Dis 36, 1221-1228.

Ribes, J. A., Vanover-Sams, C. L. \& Baker, D. J. (2000). Zygomycetes in human disease. Clin Microbiol Rev 13, 236-301.

Roilides, E., Dignani, M. C., Anaissie, E. J. \& Rex, J. H. (1998). The role of immunoreconstitution in the management of refractory opportunistic fungal infections. Med Mycol 36, 12-25.

Rubin, M. A., Carroll, K. C. \& Cahill, B. C. (2002). Caspofungin in combination with itraconazole for the treatment of invasive aspergillosis in humans. Clin Infect Dis 34, 1160-1161.

Saag, M. S., Cloud, G. A., Graybill, J. R. \& 11 other authors, and the National Institute of Allergy and Infectious Diseases Mycoses Study Group (1999). A comparison of itraconazole versus fluconazole as maintenance therapy for AIDS-associated cryptococcal meningitis. Clin Infect Dis 28, 291-296.

Saag, M. S., Graybill, R. J., Larsen, R. A., Pappas, P. G., Perfect, J. R., Powderly, W. G., Sobel, J. D. \& Dismukes, W. E., for the Mycoses Study Group Cryptococcal Subproject (2000). Practice guidelines for the management of cryptococcal disease. Clin Infect Dis 30, $710-718$.

Schwartz, S., Behre, G., Heinemann, V. \& 12 other authors (1999). Aerosolized amphotericin B inhalations as prophylaxis of invasive aspergillus infections during prolonged neutropenia: results of a prospective randomized multicenter trial. Blood 93, 3654-3661.

Singh, N., Avery, R. K., Munoz, P. \& 10 other authors (2003). Trends in risk profiles for and mortality associated with invasive aspergillosis among liver transplant recipients. Clin Infect Dis 36, 46-52.

Slain, D., Rogers, P. D., Cleary, J. D. \& Chapman, S. W. (2001). Intravenous itraconazole. Ann Pharmacother 35, 720-729. 
Slavin, M. A., Osborne, B., Adams, R., Levenstein, M. J., Schoch, H. G., Feldman, A. R., Meyers, J. D. \& Bowden, R. A. (1995). Efficacy and safety of fluconazole prophylaxis for fungal infections after marrow transplantation - a prospective, randomized, double-blind study. J Infect Dis 171, 1545-1552.

Steinbach, W. J., Stevens, D. A. \& Denning, D. W. (2003). Combination and sequential antifungal therapy for invasive aspergillosis: review of published in vitro and in vivo interactions and 6281 clinical cases from 1966 to 2001. Clin Infect Dis 37, S188-S224.

Stevens, D. A., Kan, V. L., Judson, M. A. \& 7 other authors (2000). Practice guidelines for diseases caused by Aspergillus. Clin Infect Dis 30, 696-709.

Sun, Q. N., Fothergill, A. W., McCarthy, D. I., Rinaldi, M. G. \& Graybill, J. R. (2002). In vitro activities of posaconazole, itraconazole, voriconazole, amphotericin $\mathrm{B}$, and fluconazole against 37 clinical isolates of zygomycetes. Antimicrob Agents Chemother 46, 1581-1582.

Tobon, A. M., Arango, M., Fernandez, D. \& Restrepo, A. (2003). Mucormycosis (zygomycosis) in a heart-kidney transplant recipient: recovery after posaconazole therapy. Clin Infect Dis 36, 1488-1491.

Torres, H. A., Hachem, R. Y., Chemaly, R. F., Kontoyiannis, D. P. \& Raad, II (2005). Posaconazole: a broad-spectrum triazole antifungal. Lancet Infect Dis 5, 775-785.

Tortorano, A. M., Biraghi, E., Astolfi, A. \& 8 other authors, and the FIMUA Candidemia Study Group (2002). European Confederation of Medical Mycology (ECMM) prospective survey of candidaemia: report from one Italian region. J Hosp Infect 51, 297-304.

Tortorano, A. M., Caspani, L., Rigoni, A. L., Biraghi, E., Sicignano, A. \& Viviani, M. A. (2004a). Candidosis in the intensive care unit: a 20-year survey. J Hosp Infect 57, 8-13.

Tortorano, A. M., Peman, J., Bernhardt, H. \& 8 other authors, and the ECMM Working Group on Candidaemia (2004b). Epidemiology of candidaemia in Europe: results of 28-month European Confederation of Medical Mycology (ECMM) hospital-based surveillance study. Eur J Clin Microbiol Infect Dis 23, 317-322.

Trick, W. E., Fridkin, S. K., Edwards, J. R., Hajjeh, R. A. \& Gaynes, R. P., and the National Nosocomial Infections Surveillance System Hospitals (2002). Secular trend of hospital-acquired candidemia among intensive care unit patients in the United States during 1989-1999. Clin Infect Dis 35, 627-630.

Vardakas, K. Z., Michalopoulos, A. \& Falagas, M. E. (2005). Fluconazole versus itraconazole for antifungal prophylaxis in neutropenic patients with haematological malignancies: a metaanalysis of randomised-controlled trials. Br J Haematol 131, 22-28.

Vermes, A., Guchelaar, H. J. \& Dankert, J. (2000). Flucytosine: a review of its pharmacology, clinical indications, pharmacokinetics, toxicity and drug interactions. J Antimicrob Chemother 46, 171-179.

Villanueva, A., Arathoon, E. G., Gotuzzo, E., Berman, R. S., DiNubile, M. J. \& Sable, C. A. (2001). A randomized double-blind study of caspofungin versus amphotericin for the treatment of candidal esophagitis. Clin Infect Dis 33, 1529-1535.

Villanueva, A., Gotuzzo, E., Arathoon, E. G., Noriega, L. M., Kartsonis, N. A., Lupinacci, R. J., Smietana, J. M., DiNubile, M. J. \& Sable, C. A. (2002). A randomized double-blind study of caspofungin versus fluconazole for the treatment of esophageal candidiasis. Am J Med 113, 294-299.

Walsh, T. J., Finberg, R. W., Arndt, C. \& 9 other authors (1999). Liposomal amphotericin B for empirical therapy in patients with persistent fever and neutropenia. National Institute of Allergy and Infectious Diseases Mycoses Study Group. N Engl J Med 340, 764-771.

Walsh, T. J., Pappas, P., Winston, D. J. \& 20 other authors, for the National Institute of Allergy and Infectious Diseases Mycoses Study Group (2002). Voriconazole compared with liposomal amphotericin B for empirical antifungal therapy in patients with neutropenia and persistent fever. $N$ Engl J Med 346, 225-234.

Walsh, T. J., Teppler, H., Donowitz, G. R. \& 8 other authors (2004). Caspofungin versus liposomal amphotericin B for empirical antifungal therapy in patients with persistent fever and neutropenia. $N$ Engl J Med 351, 1391-1402.

White, M. H., Bowden, R. A., Sandler, E. S. \& 9 other authors (1998). Randomized, double-blind clinical trial of amphotericin $B$ colloidal dispersion vs. amphotericin B in the empirical treatment of fever and neutropenia. Clin Infect Dis 27, 296-302.

Wingard, J. R., White, M. H., Anaissie, E., Raffalli, J., Goodman, J. \& Arrieta, A., and the $\mathrm{L}$ Amph/ABLC Collaborative Study Group (2000). A randomized, double-blind comparative trial evaluating the safety of liposomal amphotericin B versus amphotericin B lipid complex in the empirical treatment of febrile neutropenia. Clin Infect Dis 31, 1155-1163.

Winston, D. J., Pakrasi, A. \& Busuttil, R. W. (1999). Prophylactic fluconazole in liver transplant recipients. A randomized, doubleblind, placebo-controlled trial. Ann Intern Med 131, 729-737. 